\title{
Limits on anomalous neutral current triple gauge boson couplings
}

\author{
Alexander $\mathrm{Oh}^{*}$ on behalf of the LEP/TGC Working Group \\ EP Division, CERN, CH-1211 Geneve 23, Suisse \\ E-mail: 'Alexander. Oh@icern.ch'
}

ABSTRACT: The coupling of three neutral gauge bosons have been measured at LEP2with the ALEPH, DELPHI, L3 and OPAL detectors. Limits the anomalous couplings $h_{i}^{V}$ and $f_{i}^{V}$ with $i=1 . .5$ and $V=\gamma, \mathrm{Z}$ have been derived for the combined data set of all four detectors. No signs of existence of anomalous neutral triple gauge couplings have been found. For the first time off-shell couplings have been studied with the DELPHI detector.

\section{Introduction}

There are two classes of Lorentz invariant structures associated with neutral TGC vertices

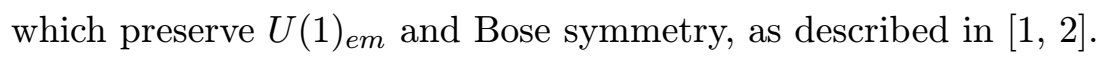

The first class refers to anomalous $\mathrm{Z} \gamma \gamma^{*}$ and $\mathrm{Z} \gamma \mathrm{Z}^{*}$ couplings which are accessible at LEP in the process $\mathrm{e}^{+} \mathrm{e}^{-} \rightarrow \mathrm{Z} \gamma$. The parametrisation contains eight couplings: $h_{i}^{V}$ with $i=1, \ldots, 4$ and $V=\gamma$, Z. The superscript $\gamma$ refers to $\mathrm{Z} \gamma \gamma^{*}$ couplings and superscript $\mathrm{Z}$ refers to $\mathrm{Z} \gamma \mathrm{Z}^{*}$ couplings. The photon and the $\mathrm{Z}$ boson in the final state are considered as on-shell particles, while the third boson at the vertex is off shell. The couplings $h_{1}^{V}$ and $h_{2}^{V}$ are CP-odd while $h_{3}^{V}$ and $h_{4}^{V}$ are CP-even.

The second class refers to anomalous $\mathrm{ZZ}^{*}$ and $\mathrm{ZZZ}^{*}$ couplings which are accessible at LEP2 in the process $\mathrm{e}^{+} \mathrm{e}^{-} \rightarrow$ ZZ. This anomalous vertex is parametrised in terms of four couplings: $f_{i}^{V}$ with $i=4,5$ and $V=\gamma$, Z. The superscript $\gamma$ refers to $\mathrm{ZZ}^{*}$ couplings and the superscript $\mathrm{Z}$ refers to $\mathrm{ZZZ}^{*}$ couplings, respectively. Both $\mathrm{Z}$ bosons in the final state are assumed to be on-shell, while the third boson at the triple vertex is off-shell. The couplings $f_{4}^{V}$ are CP-odd whereas $f_{5}^{V}$ are CP-even.

Note that the $h_{i}^{V}$ and $f_{i}^{V}$ couplings are independent of each other. They are assumed to be real and they vanish at tree level in the Standard Model.

${ }^{*}$ Speaker. 


\section{Measurements}

All experiments have measured the $h$ and $f$-couplings for all or part of the data set. The $h$-coupling analyses of ALEPH, DELPHI and L3 use the data collected at LEP2 up to centre-of-mass energies of $209 \mathrm{GeV}$. The OPAL measurements so far use the data at $189 \mathrm{GeV}$. The results of the $f$-couplings are now obtained from the whole data set above the ZZ-production threshold by all of the experiments. The experiments already pre-combine different processes and final states for each of the couplings. The analyses use measurements of the total cross sections of $\mathrm{Z} \gamma$ and $\mathrm{ZZ}$ production and the differential distributions, while L3 uses the Optimal Observables technique to extract the couplings. The individual

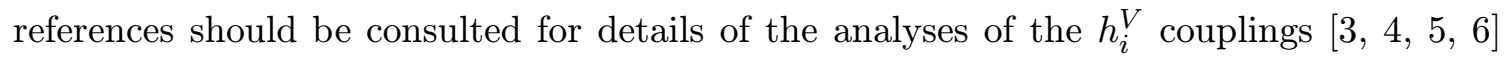

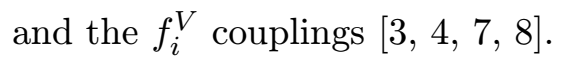

\section{Results}

Results from the four LEP experiments on the various electroweak gauge boson couplings, and their combination are presented. The results quoted for each individual experiment are calculated using the method described in 9.1 . Thus they may differ slightly from those reported in the individual references.

\subsection{Neutral Triple Gauge Boson Couplings in $\mathrm{Z} \gamma$ Production}

The individual analyses and results of the experiments for the $h$-couplings are described

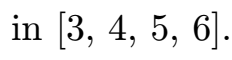

\section{Single-Parameter Analyses}

The results for each experiment are shown in Table tistical and systematic uncertainties. The results of the combination are given in Table 2.2 . The sensitivity of the L3 analysis is is the highest amongst the LEP experiments. This is partially due to the use of a larger phase space region, which increases the statistics by about a factor two, and partially due to added information from using an Optimal Observable technique.

\section{Two-Parameter Analyses}

The LEP average values are given in Table the combination.

\subsection{Neutral Triple Gauge Boson Couplings in ZZ Production}

The individual analyses and results of the experiments for the $f$-couplings are described

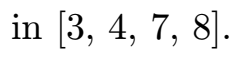

\section{Single-Parameter Analyses}

The results for each experiment are shown in Table where the errors include both statistical and systematic uncertainties. The results of the combination are given in Table 


\section{Two-Parameter Analyses}

The LEP average values are given in Table $\overline{6}$.

\subsection{Off-shell Triple Gauge Boson Couplings}

An analysis of off-shell neutral gauge boson coupling parameters from four fermion final states was presented by DELPHI. It uses the $\mu^{+} \mu^{-} q \bar{q}$ four fermion final state from $Z Z$ and $Z \gamma^{*}$ production to derive limits on anomalous coupling parameters $l_{i}^{V^{*} V^{*} V^{*}}$ with $i=1 . .3$ and $V=\gamma, Z$. No significant deviation from the Standard Model value was found and limits were set [落]

\section{Conclusions}

No significant deviation from the Standard Model prediction is seen for any of the electroweak gauge boson couplings studied.

\section{Acknowledgements}

We would like to thank the CERN accelerator divisions for the efficient operation of the LEP accelerator and the LEP gauge coupling working group for the combination of the results from the individual experiments.

\section{References}

[1] K. Hagiwara et al., Nucl. Phys. B282 (1987) 253

[2] G. J. Gounaris, J. Layssac, and F. M. Renard, Phys. Rev. D62 (2000) 073013

[3] The ALEPH Collaboration, Limits on anomalous neutral gauge couplings using data from ZZ and $Z \gamma$ production between 183-208 GeV, ALEPH 2001-061 (July 2001) CONF 2001-041.

[4] The DELPHI Collaboration, Study of Trilinear Gauge Boson Couplings $Z Z Z, Z Z \gamma$ and $Z \gamma \gamma$, DELPHI 2001-097 (July 2001) CONF 525.

[5] The L3 Collaboration, M. Acciari et al., Phys. Lett. B 436 (1999) 187; The L3 Collaboration, M. Acciari et al., Phys. Lett. B 489 (2000) 55.

The L3 Collaboration, Search for anomalous ZZg and Zgg couplings in the process ee $\rightarrow Z g$ at LEP, L3 Note 2672 (July 2001).

[6] The OPAL Collaboration, G. Abbiendi et al., Eur. Phys. J. C 17 (2000) 13.

[7] The L3 Collaboration, M. Acciari et al., Phys. Lett. B 450 (1999) 281; The L3 Collaboration, M. Acciari et al., Phys. Lett. B 465 (1999) 363; The L3 Collaboration, Z Boson Pair-Production at LEP, L3 Note 2696 (July 2001).

[8] The OPAL Collaboration, G. Abbiendi et al., Phys. Lett. B476 (2000) 256; The OPAL Collaboration, Study of $Z$ Pair Production and Anomalous Couplings in $e^{+} e^{-}$ Collisions at $\sqrt{s}$ between 190 and $209 \mathrm{GeV}$, OPAL PN482 (July 2001).

[9] The LEP-GC Working Group, LEPEWWG/TGC/2001-03, September 2001. 


\begin{tabular}{|l||r|r|r|r|}
\hline Parameter & ALEPH & DELPHI & L3 & OPAL \\
\hline \hline$h_{1}^{\gamma}$ & {$\left[\begin{array}{ll}-0.14, & +0.14\end{array}\right]$} & {$\left[\begin{array}{ll}-0.15, & +0.15\end{array}\right]$} & {$\left[\begin{array}{ll}-0.06, & +0.06\end{array}\right]$} & {$\left[\begin{array}{ll}-0.13, & +0.13\end{array}\right]$} \\
\hline$h_{2}^{\gamma}$ & {$\left[\begin{array}{ll}-0.07, & +0.07\end{array}\right]$} & {$\left[\begin{array}{ll}-0.09, & +0.09\end{array}\right]$} & {$\left[\begin{array}{ll}-0.053, & +0.024\end{array}\right]$} & {$\left[\begin{array}{ll}-0.089, & +0.089\end{array}\right]$} \\
\hline$h_{3}^{\gamma}$ & {$\left[\begin{array}{ll}-0.069, & +0.037\end{array}\right]$} & {$\left[\begin{array}{ll}-0.047, & +0.047\end{array}\right]$} & {$\left[\begin{array}{ll}-0.062, & -0.014\end{array}\right]$} & {$\left[\begin{array}{ll}-0.16, & +0.00\end{array}\right]$} \\
\hline$h_{4}^{\gamma}$ & {$\left[\begin{array}{ll}-0.020, & +0.045\end{array}\right]$} & {$\left[\begin{array}{ll}-0.032, & +0.030\end{array}\right]$} & {$\left[\begin{array}{ll}-0.004, & +0.045\end{array}\right]$} & {$\left[\begin{array}{ll}+0.01, & +0.13\end{array}\right]$} \\
\hline$h_{1}^{Z}$ & {$\left[\begin{array}{ll}-0.23, & +0.23\end{array}\right]$} & {$\left[\begin{array}{ll}-0.24, & +0.25\end{array}\right]$} & {$\left[\begin{array}{ll}-0.17, & +0.16\end{array}\right]$} & {$\left[\begin{array}{ll}-0.22, & +0.22\end{array}\right]$} \\
\hline$h_{2}^{Z}$ & {$\left[\begin{array}{ll}-0.12, & +0.12\end{array}\right]$} & {$\left[\begin{array}{ll}-0.14, & +0.14\end{array}\right]$} & {$\left[\begin{array}{ll}-0.10, & +0.09\end{array}\right]$} & {$\left[\begin{array}{ll}-0.15, & +0.15\end{array}\right]$} \\
\hline$h_{3}^{Z}$ & {$\left[\begin{array}{ll}-0.28, & +0.19\end{array}\right]$} & {$\left[\begin{array}{ll}-0.32, & +0.18\end{array}\right]$} & {$\left[\begin{array}{ll}-0.23, & +0.11\end{array}\right]$} & {$\left[\begin{array}{ll}-0.29, & +0.14\end{array}\right]$} \\
\hline$h_{4}^{Z}$ & {$\left[\begin{array}{ll}-0.10, & +0.15\end{array}\right]$} & {$\left[\begin{array}{ll}-0.12, & +0.18\end{array}\right]$} & {$\left[\begin{array}{ll}-0.08, & +0.16\end{array}\right]$} & {$\left[\begin{array}{ll}-0.09, & +0.19\end{array}\right]$} \\
\hline
\end{tabular}

Table 1: The $95 \%$ C.L. intervals $(\Delta \log \mathcal{L}=1.92)$ measured by the ALEPH, DELPHI, L3 and OPAL. In each case the parameter listed is varied while the remaining ones are fixed to their Standard Model values. Both statistical and systematic uncertainties are included.

\begin{tabular}{|l||c|}
\hline Parameter & $95 \%$ C.L. \\
\hline \hline$h_{1}^{\gamma}$ & {$\left[\begin{array}{ll}-0.056, & +0.055\end{array}\right]$} \\
\hline$h_{2}^{\gamma}$ & {$\left[\begin{array}{ll}-0.045, & +0.025\end{array}\right]$} \\
\hline$h_{3}^{\gamma}$ & {$\left[\begin{array}{ll}-0.049, & -0.008\end{array}\right]$} \\
\hline$h_{4}^{\gamma}$ & {$\left[\begin{array}{ll}-0.002, & +0.034\end{array}\right]$} \\
\hline$h_{1}^{Z}$ & {$\left[\begin{array}{ll}-0.13, & +0.13\end{array}\right]$} \\
\hline$h_{2}^{Z}$ & {$\left[\begin{array}{ll}-0.078, & +0.071\end{array}\right]$} \\
\hline$h_{3}^{Z}$ & {$\left[\begin{array}{ll}-0.20, & +0.07\end{array}\right]$} \\
\hline$h_{4}^{Z}$ & {$\left[\begin{array}{ll}-0.05, & +0.12\end{array}\right]$} \\
\hline
\end{tabular}

Table 2: The $95 \%$ C.L. intervals $(\Delta \log \mathcal{L}=1.92)$ obtained combining the results from the four experiments. In each case the parameter listed is varied while the remaining ones are fixed to their Standard Model values. Both statistical and systematic uncertainties are included. 


\begin{tabular}{|c|c|c|c|c|}
\hline Parameter & \multicolumn{2}{|c|}{ 95\% C.L. } & \multicolumn{2}{|c|}{ Correlations } \\
\hline$h_{1}^{\gamma}$ & {$[-0.16$} & $+0.05]$ & 1.00 & +0.79 \\
\hline$h_{2}^{\gamma}$ & {$[-0.11$} & $+0.02]$ & +0.79 & 1.00 \\
\hline$h_{3}^{\gamma}$ & {$[-0.08$} & $+0.14]$ & 1.00 & +0.97 \\
\hline$h_{4}^{\gamma}$ & {$[-0.04$} & $+0.11]$ & +0.97 & 1.00 \\
\hline$h_{1}^{Z}$ & {$[-0.35$} & $+0.28]$ & 1.00 & +0.77 \\
\hline$h_{2}^{Z}$ & {$[-0.21$} & $+0.17]$ & +0.77 & 1.00 \\
\hline$h_{3}^{Z}$ & {$[-0.37$} & $+0.29]$ & 1.00 & +0.76 \\
\hline$h_{4}^{Z}$ & {$[-0.19$} & $+0.21]$ & +0.76 & 1.00 \\
\hline
\end{tabular}

Table 3: The $95 \%$ C.L. intervals $(\Delta \log \mathcal{L}=1.92)$ obtained combining the results from ALEPH, DELPHI and L3. In each case the two parameters listed are varied while the remaining ones are fixed to their Standard Model values. Both statistical and systematic uncertainties are included. Since the shape of the log-likelihood is not parabolic, there is some ambiguity in the definition of the correlation coefficients and the values quoted here are approximate.

\begin{tabular}{|l||rl|r|r|r|}
\hline Parameter & ALEPH & DELPHI & L3 & OPAL \\
\hline \hline$f_{4}^{\gamma}$ & {$\left[\begin{array}{ll}-0.26, & +0.26\end{array}\right]$} & {$\left[\begin{array}{ll}-0.26, & +0.28\end{array}\right]$} & {$\left[\begin{array}{ll}-0.24, & +0.26\end{array}\right]$} & {$\left[\begin{array}{ll}-0.36, & +0.36\end{array}\right]$} \\
\hline$f_{4}^{Z}$ & {$\left[\begin{array}{ll}-0.44, & +0.43\end{array}\right]$} & {$\left[\begin{array}{ll}-0.49, & +0.42\end{array}\right]$} & {$\left[\begin{array}{ll}-0.43, & +0.41\end{array}\right]$} & {$\left[\begin{array}{ll}-0.55, & +0.64\end{array}\right]$} \\
\hline$f_{5}^{\gamma}$ & {$\left[\begin{array}{ll}-0.54, & +0.56\end{array}\right]$} & {$\left[\begin{array}{ll}-0.48, & +0.61\end{array}\right]$} & {$\left[\begin{array}{ll}-0.48, & +0.56\end{array}\right]$} & {$\left[\begin{array}{ll}-0.82, & +0.72\end{array}\right]$} \\
\hline$f_{5}^{Z}$ & {$\left[\begin{array}{ll}-0.73, & +0.83\end{array}\right]$} & {$\left[\begin{array}{ll}-0.42, & +0.69\end{array}\right]$} & {$\left[\begin{array}{ll}-0.46, & +1.2\end{array}\right]$} & {$\left[\begin{array}{ll}-0.96, & +0.31\end{array}\right]$} \\
\hline
\end{tabular}

Table 4: The $95 \%$ C.L. intervals $(\Delta \log \mathcal{L}=1.92)$ measured by ALEPH, DELPHI, L3 and OPAL. In each case the parameter listed is varied while the remaining ones are fixed to their Standard Model values. Both statistical and systematic uncertainties are included.

\begin{tabular}{|l||c|}
\hline Parameter & $95 \%$ C.L. \\
\hline \hline$f_{4}^{\gamma}$ & {$\left[\begin{array}{ll}-0.17, & +0.19\end{array}\right]$} \\
\hline$f_{4}^{Z}$ & {$\left[\begin{array}{ll}-0.31, & +0.28\end{array}\right]$} \\
\hline$f_{5}^{\gamma}$ & {$\left[\begin{array}{ll}-0.36, & +0.40\end{array}\right]$} \\
\hline$f_{5}^{Z}$ & {$\left[\begin{array}{ll}-0.36, & +0.39\end{array}\right]$} \\
\hline
\end{tabular}

Table 5: The $95 \%$ C.L. intervals $(\Delta \log \mathcal{L}=1.92)$ obtained combining the results from all four experiments. In each case the parameter listed is varied while the remaining ones are fixed to their Standard Model values. Both statistical and systematic uncertainties are included. 


\begin{tabular}{|l||c|rr|}
\hline Parameter & 95\% C.L. & \multicolumn{2}{|c|}{ Correlations } \\
\hline \hline$f_{4}^{\gamma}$ & {$\left[\begin{array}{ll}-0.17, & +0.19\end{array}\right]$} & 1.00 & +0.10 \\
$f_{4}^{Z}$ & {$\left[\begin{array}{ll}-0.30, & +0.28\end{array}\right]$} & +0.10 & 1.00 \\
\hline$f_{5}^{\gamma}$ & {$\left[\begin{array}{ll}-0.34, & +0.38\end{array}\right]$} & 1.00 & -0.18 \\
$f_{5}^{Z}$ & {$\left[\begin{array}{ll}-0.36, & +0.38\end{array}\right]$} & -0.18 & 1.00 \\
\hline
\end{tabular}

Table 6: The $95 \%$ C.L. intervals $(\Delta \log \mathcal{L}=1.92)$ obtained combining the results from all four experiments. In each case the two parameters listed are varied while the remaining ones are fixed to their Standard Model values. Both statistical and systematic uncertainties are included. Since the shape of the log-likelihood is not parabolic, there is some ambiguity in the definition of the correlation coefficients and the values quoted here are approximate. 